\title{
Article \\ Radiation Dose and Fluoroscopy Time of Endovascular Coil Embolization in Patients with Carotid Cavernous Fistulas
}

\author{
Yigit Ozpeynirci *, Christoph Gregor Trumm (D, Robert Stahl (D), Thomas Liebig and Robert Forbrig
}

Citation: Ozpeynirci, Y.; Trumm, C.G.; Stahl, R.; Liebig, T.; Forbrig, R. Radiation Dose and Fluoroscopy

Time of Endovascular Coil

Embolization in Patients with

Carotid Cavernous Fistulas.

Diagnostics 2022, 12, 531. https:// doi.org/10.3390/diagnostics12020531

Academic Editor: Ernesto Di Cesare

Received: 25 January 2022

Accepted: 17 February 2022

Published: 18 February 2022

Publisher's Note: MDPI stays neutral with regard to jurisdictional claims in published maps and institutional affiliations.

Copyright: (C) 2022 by the authors. Licensee MDPI, Basel, Switzerland. This article is an open access article distributed under the terms and conditions of the Creative Commons Attribution (CC BY) license (https:// creativecommons.org/licenses/by/ $4.0 /)$.
Institute of Neuroradiology, University Hospital, LMU Munich, Marchioninistr. 15, 81377 Munich, Germany; christoph.trumm@med.uni-muenchen.de (C.G.T.); robert.stahl@med.uni-muenchen.de (R.S.); thomas.liebig@med.uni-muenchen.de (T.L.); robert.forbrig@med.uni-muenchen.de (R.F.)

* Correspondence: yigit.oezpeynirci@med.uni-muenchen.de
Abstract: Carotid cavernous fistulas (CCFs) are abnormal connections between the cavernous sinus and the internal and/or external carotid artery. Endovascular therapy is the gold standard treatment. In the current retrospective single-center study we report detailed dosimetrics of all patients with CCFs treated by endovascular coil embolization between January 2012 and August 2021. Procedural and dosimetric data were compared between direct and indirect fistulas according to Barrow et al., and different DSA protocol groups. The local diagnostic reference level (DRL) was defined as the 3rd quartile of the dose distribution. In total, thirty patients met the study criteria. The local DRL was $376.2 \mathrm{~Gy} \mathrm{~cm}^{2}$. The procedural dose area product (DAP) $(p=0.03)$ and the number of implanted coils $(p=0.02)$ were significantly lower in direct fistulas. The median values for fluoroscopy time (FT) $(p=0.08)$ and number of DSA acquisitions $(p=0.84)$ were not significantly different between groups. There was a significantly positive correlation between DAP and FT ( $p=0.003)$. The application of a dedicated low-dose protocol yielded a $32.6 \%$ DAP reduction. In conclusion, this study provides novel DRLs for endovascular CCF treatment using detachable coils. The data presented in this work might be used to establish new specific DRLs.

Keywords: arteriovenous fistula; cerebral angiography; radiation exposure; endovascular procedure; cerebrovascular disorders

\section{Introduction}

Carotid cavernous fistulas (CCFs) are abnormal arteriovenous (AV) connections between the cavernous sinus (CS) and the internal carotid artery (ICA) or dural branches of the ICA and/or external carotid artery (ECA) [1-3].

Exophthalmos, retroorbital pain, chemosis, ophthalmoplegia, and fast decline of visual acuity, eventually leading to permanent vision loss, are symptoms of a CCF. Furthermore, CCF-induced reflux into the cortical cerebral veins (which normally drain into the CS) may yield venous congestion and intracranial hemorrhage [2,3].

Barrow et al., introduced the most commonly used classification, which categorizes CCFs into two main groups (direct and indirect) and four subgroups (Type A-D) [1] Direct fistulas (Type A) represent pathological AV communications between the ICA and the CS. They are usually caused by laceration of the cavernous ICA segment following a severe head trauma with or without a concomitant skull base fracture, rupture of a cavernous ICA aneurysm, genetic connective tissue disorders (e.g., Ehlers-Danlos syndrome or fibromuscular dysplasia) and/or iatrogenic injuries (e.g., surgical trauma). Direct CCFs usually require emergent treatment because of their high-flow nature, acute presentation and the possibility of rapid deterioration. In contrast, indirect fistulas (Type B-D) are characterized by uni- or bilateral connections between the dural branches of the ICA (Barrow Type B), ECA (Type C), or both arteries (Type D) and the CS. The causes of indirect CCFs are unknown, but reported predisposing factors are pregnancy, sinusitis, trauma, 
CS thrombosis, surgery, female gender and age $>50$ years. Indirect CCFs typically yield a low-flow AV shunt with gradually progressive and subacute symptoms [1-3].

Treatment methods consist of microneurosurgery, stereotactic radiosurgery and endovascular embolization. In recent years, fluoroscopically-guided endovascular therapy has become the gold standard treatment of CCF, as this technique allows for higher and faster cure rates as well as lower procedure-related morbidity when compared to other methods [3-9]. In particular, previous studies have shown that endovascular embolization through a transvenous route with detachable coils is considered the most effective treatment of CCF [6-9]. The favorite route to the CS described in the literature is through the inferior petrosal sinus [6]. In a recent meta-analysis consisting of 22 studies reporting 1066 procedures in 1043 patients with indirect CCFs, Alexandre et al., showed that transvenous coiling was the most common treatment approach (712/1066, 57.8\%), yielding both high radiological and clinical success as well as low complication rates [10].

However, as for every procedure involving radiation exposure to both the patient and the physician, diagnostic reference levels (DRL) are needed as a quality improvement tool and to maintain diagnostic and therapeutic standards. Regarding neuroendovascular procedures, the current national guidelines for radiation protection, updated in 2018 [11], define national DRLs only for mechanical thrombectomy and embolization of an intracranial aneurysm [12]. In recent publications of our research group, local DRLs for endovascular treatment of unruptured intracranial aneurysms and intracranial lateral dural arteriovenous fistulas (DAVF), as well as for diagnostic angiographies in patients with spinal DAVFs, were defined [13-15] in order to expand the neuroendovascular DRL collection. In the current monocentric study, detailed dosimetrics in CCF patients treated by fluoroscopically-guided coil embolization are presented and compared between patients with direct and indirect CCFs, as well as between various DSA acquisition protocols. These data might be valuable in order to introduce novel dedicated DRLs, as only a few dosimetry articles specifically dealing with the endovascular treatment of this subtype of intracranial fistulas have been published so far [16].

\section{Materials and Methods}

This retrospective single-centre study was approved by the responsible Institutional Review Board of the Ludwig-Maximilians-University Munich, Germany (project number 20-450). A retrospective data analysis from all consecutive patients who presented with a CCF treated at our institution between January 2012 and August 2021 was performed. The following inclusion and exclusion criteria were set:

Inclusion criteria:

- $\quad$ Age $\geq 18$ years

- $\quad$ Presence of a fistula between the ICA and/or ECA and the CS

- Treatment with endovascular embolization using only detachable coils

Exclusion criteria:

- CCFs treated with alternative endovascular methods (e.g., detachable balloons, liquid embolic agents, covered stents, flow-diverter stents or combined techniques)

- Recurrent fistulas

- $\quad$ Fistulas located elsewhere but also treated with CS coil embolization

\subsection{Procedure}

All endovascular procedures were performed under general anesthesia using a biplane angiographic unit (Axiom Artis Zee, Siemens Healthineers, Erlangen, Germany) by six experienced neuroradiologists with between five and more than 20 years of experience in neurointerventions. In all patients, arterial and/or venous access was achieved using a transfemoral approach. The non-ionic iodinated contrast agent applied was Iomeprol $300 \mathrm{mg}$ iodine/mL (Imeron, Bracco Imaging, Kontanz, Germany). Regarding patients treated by a transarterial approach, after placing a guiding catheter in the target ICA, the 
venous side of the fistula was catheterized with a 0.017-inch microcatheter through the tear in the ICA wall. In patients treated by a transvenous approach, the microcatheter was advanced through the inferior petrosal sinus into the fistulous CS compartment. In both methods, the fistulous CS part was filled using detachable platinum coils until the AV shunt was fully closed. The initial and final diagnostic digital subtraction angiography (DSA) acquisitions on standard anteriorposterior and lateral projections were performed using a field of view (FOV) of $32 \mathrm{~cm}$ and a frame rate of 1-4 frames/second (f/s). DSA acquisitions on working projections were performed using a targeted FOV of either $11 \mathrm{~cm}$ or $16 \mathrm{~cm}$ and a higher frame rate of up to $7.5 \mathrm{f} / \mathrm{s}$. The frame rate of pulsed fluoroscopy was $7.5 \mathrm{f} / \mathrm{s}$. With respect to the DSA acquisition type, two protocols were preset by the manufacturer as previously reported [13] and applied under discretion of the treating physicians:

- $\quad$ Low-dose (LD): tube voltage $73 \mathrm{kV}$, pulse width $50 \mathrm{~ms}$, dose $1820 \mu \mathrm{Gy} / \mathrm{pulse}$

- $\quad$ Normal-dose (ND): tube voltage $73 \mathrm{kV}$, pulse width $100 \mathrm{~ms}$, dose $3000 \mu \mathrm{Gy} /$ pulse

In mixed-dose (MD) cases, both DSA protocols (LD and ND) were used during the same intervention. In order to examine the impact of different DSA protocols on the radiation exposure, two groups were formed: (1) LD group and (2) ND/MD group.

\subsection{Data Collection and Dosimetry Analysis}

Patient demographics (age, sex) and procedural data (fistula type and site, access route to the fistula, immediate angiographic success of the treatment and number of detached coils) were retrospectively obtained from the medical charts. Imaging data and dose reports were retrieved from a picture archiving and communication system (syngo.imaging, Siemens Healthineers). In terms of procedural radiation exposure, the following dosimetry parameters were collected: dose area product (DAP, Gy cm${ }^{2}$ ), fluoroscopy time (FT) and number of DSA acquisitions. The total DAP was calculated by adding the DAP of fluoroscopy and DSA acquisitions together.

\subsection{Statistics}

Data were initially tested for normality using the Shapiro-Wilk test. Continuous variables were presented as means \pm standard deviation (sd), percentages and ranges. Counts and percentages were calculated to represent categorical data. The local DRL for the endovascular coil embolization of CCFs was set at the third quartile value of the DAP distribution [17].

The two-sided unpaired $t$-test was applied to compare procedural DAP, FT, and number of detached coils between direct and indirect fistulas. The Mann-Whitney U test was used to compare the number of DSA acquisitions between cases with direct and indirect fistulas. The same tests were used to compare the same variables between the LD group and the ND/MD group. Furthermore, a Spearman correlation analysis was performed to determine the relationship between procedural DAP, FT, number of DSA acquisitions and implanted coils. A linear regression analysis was made between procedural DAP and FT.

All calculations were carried out using SPSS software Version 26.0 (IBM, Armonk, New York, NY, USA). A p-value < 0.05 was considered statistically significant.

\section{Results}

Between January 2012 and August 2021, 30 patients with CCFs treated by endovascular means met the inclusion and exclusion criteria. Table 1 shows patient demographics and procedural data. Causes of direct fistulas were a ruptured aneurysm of the cavernous ICA segment $(n=4)$, severe head trauma $(n=2)$, and spontaneous ICA dissection $(n=1)$. No procedure-related complications, which would have prolonged the intervention time, were documented. 
Table 1. Patient demographics and procedural data.

\begin{tabular}{lc}
\hline Mean Age, Years (Range) & $66(41-85)$ \\
Female sex & $23 / 30(77 \%)$ \\
Number of CCFs & \\
$\quad$-Direct (Type A) & $7 / 30(23 \%)$ \\
$\quad$-Indirect & $23 / 30(77 \%)$ \\
Type B & $4 / 30(13 \%)$ \\
$O$ Type C & $2 / 30(7 \%)$ \\
O Type D & $17 / 30(57 \%)$ \\
Angiographic outcome & \\
$\quad$-Total occlusion & $19 / 30(63 \%)$ \\
$\quad$-Small remnant & $11 / 30(37 \%)$ \\
Approach type & \\
$\quad$-Transarterial & $2 / 30(7 \%)$ \\
$\quad$-Transvenous & $27 / 30(90 \%)$ \\
$\quad$-Combined & $1 / 30(3 \%)$ \\
Fistula site & \\
$\quad$-Right & $9 / 30(30 \%)$ \\
$\quad$ Left & $8 / 30(27 \%)$ \\
$\quad$-Bilateral & $13 / 30(43 \%)$ \\
\hline
\end{tabular}

CCF: carotid cavernous fistula.

The local DRL was $376.2 \mathrm{~Gy} \mathrm{~cm}^{2}$. The 75th percentile of the FT distribution was $241.8 \mathrm{~min}$ (Table 2). $7 / 30$ (23\%) patients exceeded the local DRL. All of these patients had an indirect fistula.

Table 2. Distribution of radiation dose and fluoroscopy time.

\begin{tabular}{ccccccc}
\hline & & & \multicolumn{3}{c}{ Fluoroscopy Time, Minutes } \\
\hline & 25th percentile & Median & 75th percentile & 25th percentile & Median & 75th percentile \\
All $(\mathrm{n}=30)$ & 230.2 & 291.8 & 376.2 & 115.9 & 174.1 & 241.8 \\
Direct $(\mathrm{n}=7)$ & 113.7 & 264.2 & 271.2 & 95.3 & 117.6 & 194.8 \\
Indirect $(\mathrm{n}=23)$ & 240.2 & 330.5 & 428.8 & 130.9 & 176.2 & 245.8 \\
\hline
\end{tabular}

DAP: dose area product.

Regarding the fistula type, significantly lower median values were calculated in patients with direct CCFs both for procedural DAP $(p=0.03)$ and number of implanted coils $(p=0.02)$ when compared to patients with indirect CCFs. Median values for FT ( $p=0.08)$ and number of DSA acquisitions ( $p=0.84$ ) were not significantly different between groups (Figure 1, Tables 2 and 3).

Pair-wise correlations of the dosimetrics (DAP, FT, and number of DSA acquisitions) and number of implanted coils showed a significantly positive correlation only between radiation dose and FT ( $\left.p=0.003, \mathrm{R}^{2}=0.3\right)$, with a DAP increase of $0.96 \mathrm{~Gy} \mathrm{~cm}^{2}$ per additional minute of FT according to the linear regression analysis (Figure 2).

An LD DSA protocol was used in 20/30 (68\%) patients whereas an ND or MD DSA protocol was applied in 10/30 (32\%) patients. Regarding radiation exposure, the mean DAP was significantly lower in the LD group when compared to the ND/MD group (274.6 versus $407.4 \mathrm{~Gy} \mathrm{~cm}^{2}, p=0.02$ ). In contrast, no significant differences between groups were calculated for the mean FT (LD 186 versus ND/MD $179.5 \mathrm{~min}, p=0.84$ ), mean number of implanted coils (LD 22.4 versus ND/MD 20.3, $p=0.65$ ) and mean DSA acquisitions (LD 40.7 versus ND/MD 40.8, $p=0.58$ ) (Table 4 ). 


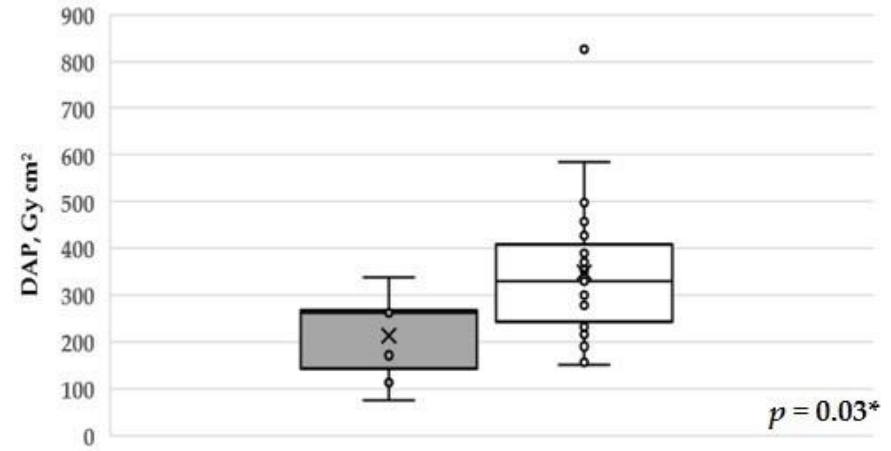

$\square$ Direct CCF (n=7) $\square$ Indirect CCF (n=23)

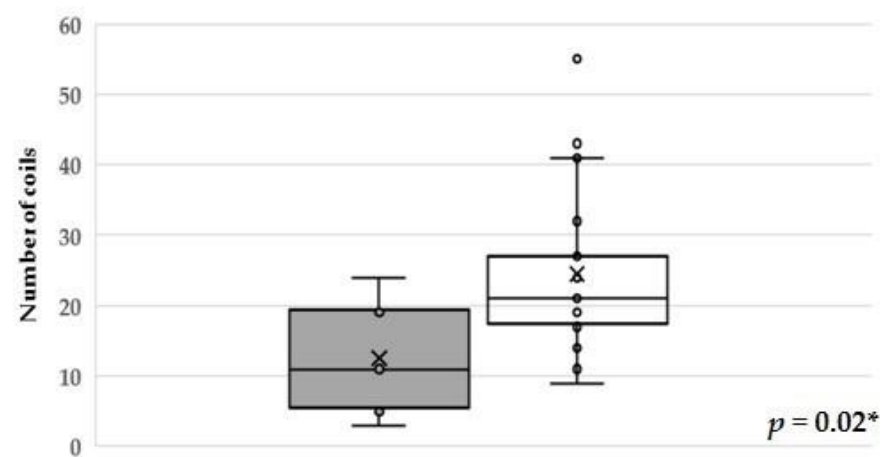

$\square$ Direct CCF (n=7) $\square$ Indirect CCF ( $=23)$

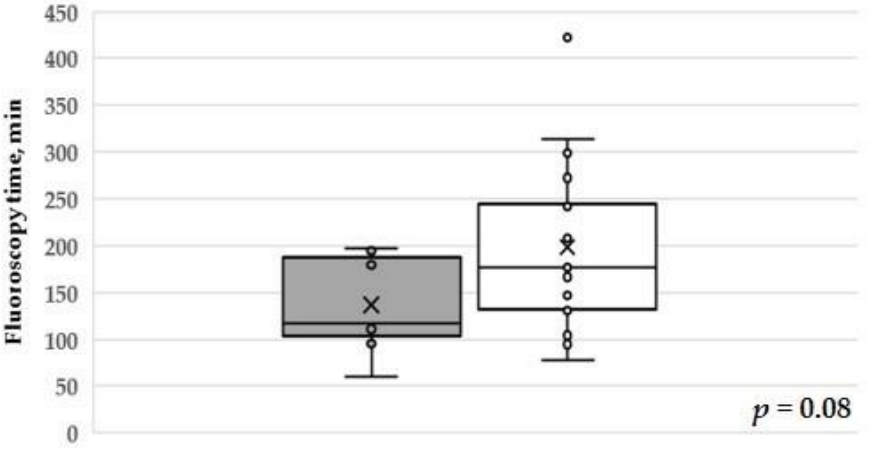

$\square$ Direct CCF (n=7) $\square$ Indirect CCF (n=23)

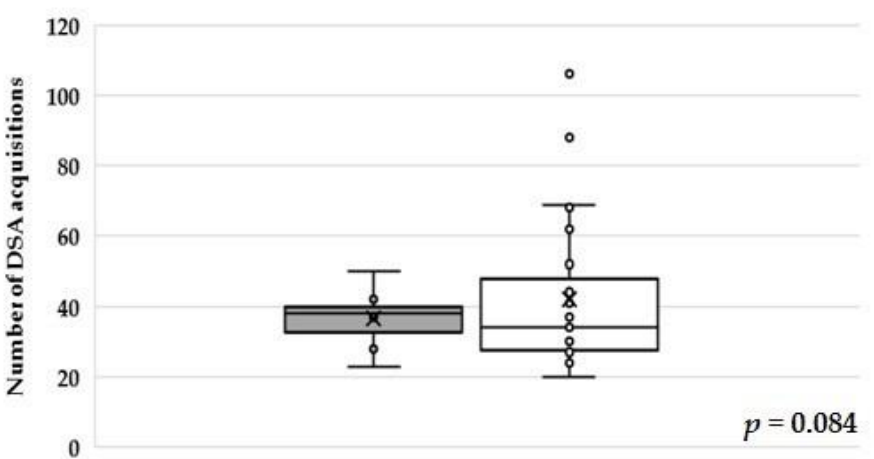

$\square$ Direct CCF (n=7) $\square$ Indirect CCF (n=23)

Figure 1. Box plots and scatter plots of total DAP, FT, number of implanted coils, and DSA acquisitions showing different types of CCFs. Significantly higher DAP $(p=0.03)$ and number of implanted coils $(p=0.02)$ in the indirect group. No significant difference in FT $(p=0.08)$ or number of DSA acquisitions $(p=0.84)$ between two groups. DAP: dose area product, FT: fluoroscopy time, DSA: digital subtraction angiography, CCF: carotid cavernous fistula * indicates statistically significant difference.

Table 3. Dosimetrics and number of implanted coils.

\begin{tabular}{|c|c|c|c|}
\hline & All CCFs $(n=30)$ & Direct CCFs $(n=7)$ & Indirect CCFs $(n=23)$ \\
\hline Mean DAP, Gy cm² (range) & $318.8 \pm 148.1(75.9-826.6)$ & $215.1 \pm 89(75.9-339.9)$ & $350.4 \pm 148.1(153-826.6)$ \\
\hline Median DAP, Gy $\mathrm{cm}^{2}$ & 291.8 & 264.2 & 330.5 \\
\hline Mean FT, min (range) & $183.8 \pm 81.4(59.4-422.2)$ & $136.4 \pm 50.2(59.4-197.7)$ & $198.3 \pm 83.6(77.8-422.2)$ \\
\hline Median FT, min & 174.1 & 117.6 & 176.2 \\
\hline Mean number of DSA runs (range) & $40.8 \pm 19.6(20-106)$ & $36.6 \pm 8.2(23-50)$ & $42 \pm 21.8(20-106)$ \\
\hline Median number of DSA runs & 35.5 & 38 & 34 \\
\hline Mean number of detached coils (range) & $21.7 \pm 11.7(3-55)$ & $12.6 \pm 7.8(3-24)$ & $24.5 \pm 11.2(9-55)$ \\
\hline Median number of detached coils & 19.5 & 11 & 21 \\
\hline
\end{tabular}

CCF: carotid cavernous fistula, DAP: dose area product, FT: fluoroscopy time. Values that are significantly different between direct and indirect CCFs are written in bold. 


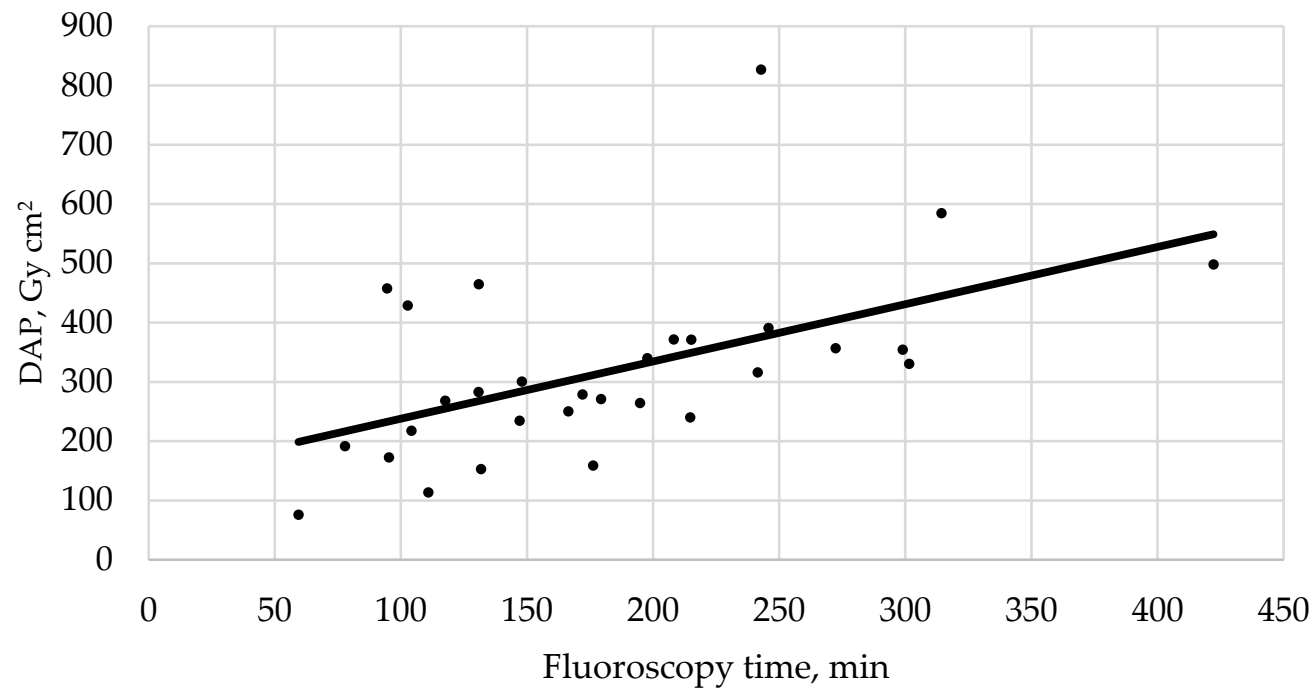

Figure 2. The relationship between fluoroscopy time and radiation exposure is summarized by the fitted regression line.

Table 4. Comparison of dosimetric data and number of implanted coils between treatment groups with different DSA protocols.

\begin{tabular}{lcccc}
\hline & All CCFs $(\mathbf{n}=\mathbf{3 0})$ & Low-Dose $(\mathbf{n}=\mathbf{2 0})$ & $\begin{array}{c}\text { Normal- and } \\
\text { Mixed-Dose }(\mathbf{n}=\mathbf{1 0})\end{array}$ & $\boldsymbol{p}$ Value \\
\hline Mean DAP, Gy cm & & $\mathbf{2 7 4 . 6}$ & $\mathbf{4 0 7 . 4}$ & $\mathbf{0 . 0 2}$ \\
Mean FT, min & 318.8 & 186.1 & 179.5 & 0.84 \\
Mean number of detached coils & 183.8 & 22.4 & 20.3 & 0.65 \\
Mean number of DSA acquisitions & 21.7 & 40.7 & 40.8 & 0.58 \\
\hline
\end{tabular}

CCF: carotid cavernous fistula, DAP: dose area product, DSA, digital subtraction angiography, FT: fluoroscopy time. Values that are significantly different are written in bold.

\section{Discussion}

International advisory organizations on ionizing radiation safety emphasize the importance of the justification of patients' exposure to radiation, as well as the need of recording the radiation dose of each examination and applying suitable DRLs [17].

A comprehensive literature search revealed no officially established or recommended DRLs for the endovascular treatment of CCFs [12,18-20]. In this retrospective, single-center study, detailed dosimetry data are reported from $30 \mathrm{CCFs}$ endovascularly treated by coil embolization. The mean procedural DAP was $318.8 \mathrm{~Gy} \mathrm{~cm}^{2}$ and the average FT was $183.8 \mathrm{~min}$. Local 3rd quartile values were $376.2 \mathrm{~Gy} \mathrm{~cm}^{2}$ for DAP (i.e., DRL) and $241.8 \mathrm{~min}$ for FT.

Only in a few dosimetry studies, have intracranial DAFVs been represented as a subcategory $[14,21,22]$. The reported results in terms of local DRLs were quite various $\left(414 \mathrm{~Gy} \mathrm{~cm}^{2}\right.$, $507.33 \mathrm{~Gy} \mathrm{~cm}^{2}$ and $730 \mathrm{~Gy} \mathrm{~cm}^{2}$ ). However, Forbrig et al. [14] and Opitz et al. [21] excluded CCFs, and Kien et al. [22] did not describe the inclusion criteria in detail. Regarding FT, Forbrig et al., proposed $142 \mathrm{~min}$ and Kien et al., $80 \mathrm{~min}$.

Intracranial DAFVs represent a heterogenous group of vascular pathologies handled in different ways using various embolic materials. As a result, local DRLs suggested by multiple neurovascular centers may differ substantially, consequently disabling adequate comparison with our findings.

In the only study published on radiation exposure in endovascular management of CCFs, Opitz et al. [16] proposed a DRL of $350 \mathrm{~Gy} \mathrm{~cm}^{2}$, which is in line with our observations. The mean FT in our study group was yet substantially higher than in their study (183.8 vs. $61.9 \mathrm{~min}$ ). One explanation for this finding might be a comparably higher number of technically challenging procedures in our study group due to a difficult vascular 
anatomy in some cases, which in turn may have required a longer time for successful catheterization of the fistula-harboring CS compartment. In this context, a longer FT alone does not necessarily imply a significantly increased radiation dose, because most of the procedural irradiation stems from DSA acquisitions but not from pulsed fluoroscopy. However, as expected, a positive correlation between FT and procedural DAP ( $p=0.003)$ was found in our study group.

Direct CCFs usually develop suddenly because of a tear on the ICA wall (e.g., after aneurysm rupture), yielding high-flow AV shunts in most cases. Thus, they are commonly diagnosed earlier than indirect fistulas. The therapeutic strategy, depending on the cause, is either transarterial treatment of the aneurysm or transvenous occlusion of the fistulous CS compartment. In contrast, indirect CCFs are commonly low-flow, chronic lesions and are accompanied by thrombotic occlusion of the draining veins, resulting in a technically more challenging CS catheterization. Furthermore, in indirect CCFs the fistulous CS compartment is commonly larger when compared to direct fistulas. Accordingly, in the present study values for both the periprocedural DAP $(p=0.03)$ and number of implanted coils $(p=0.02)$ were significantly higher in patients with indirect fistulas when compared to those with direct fistulas. Moreover, each of the seven patients exceeding our local DRL level had an indirect fistula type.

There was no correlation between the amount of coils used and FT, radiation exposure or number of DSA runs. These data outline the fact that navigation of the microcatheter towards the target (i.e., fistulous CS compartment) represents the most time-consuming part of the intervention, and that the number of subsequently detached coils does not substantially affect overall procedural radiation dose. Figure 3 illustrates two patients with indirect fistulas endovascularly treated by 9 and 55 coils, respectively. DAP values were comparable between both cases (316.1 vs. $330.5 \mathrm{~Gy} \mathrm{~cm}^{2}$ ).

Regarding radiation dose optimization in the field of interventional neuroradiology, several techniques have been proposed in recent years [13,23-26]. At our institution, neurointerventionists may choose between two preset DSA protocols (LD and ND) dependent on the patient age, vascular pathology, purpose of the examination (diagnostic or therapeutic) or required image resolution (e.g., ND during superselective catheterization of the fistula point and LD during coiling of the fistula). In this study, patients in the LD group had a significantly lower radiation exposure compared to the other group (mean DAP 274.6 versus $407.4 \mathrm{~Gy} \mathrm{~cm}^{2}, p=0.02$ ), without any significant difference regarding further procedural parameters such as FT, number of DSA acquisitions and number of implanted coils. The LD protocol yielded a dose reduction of $32.6 \%$. The positive impact of this dedicated DSA protocol on radiation dose was also demonstrated in recently published papers from our institution [13-15].

For the determination of local DRLs with acceptable 95\% confidence intervals, Miller et al., propose at least 30 studies of the same procedure [27]. Hence, the dosimetry results of our study population may substantially contribute to the literature in order to introduce novel DRLs in the field of endovascular CCF treatment. Although rare, these vascular disorders represent an important subgroup of (often time-consuming) neurointerventional procedures, thus demanding proper documentation and optimization of radiation exposure.

This study has several limitations because of the mono-centric design. Angiographies were performed using a single angiographic system from a single vendor (Siemens Healthineers). Furthermore, patients treated with liquid embolics or direct puncture of the ophthalmic vein were explicitly excluded for the sake of homogeneity, hence the provided dosimetric data cannot be generalized to the entire treatment spectrum of CCFs. However, this study still covers the majority of patients undergoing endovascular CCF treatment. 


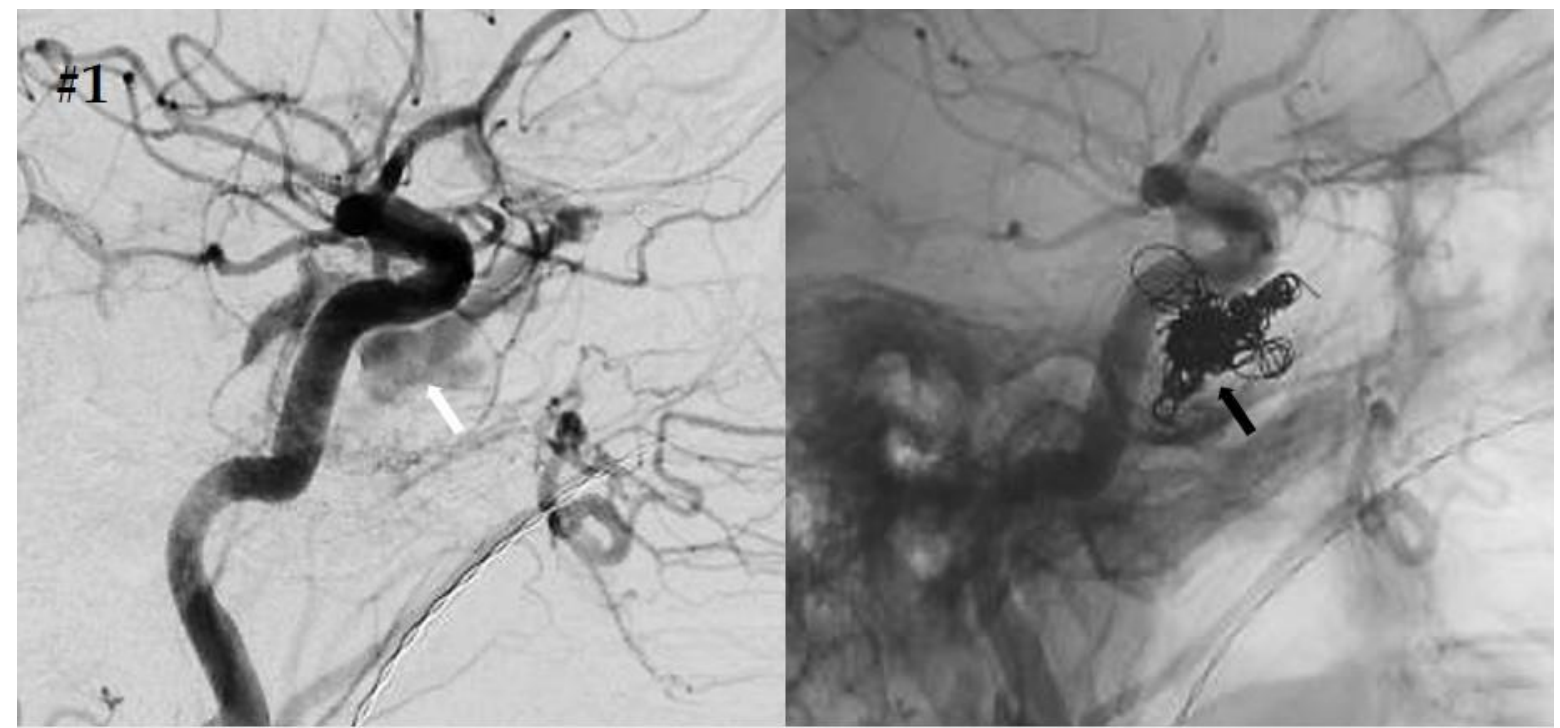

\section{9 coils, 27 acquisitions, FT: 241.5 min, DAP: $316.1 \mathrm{~Gy} \mathrm{~cm}^{2}$}

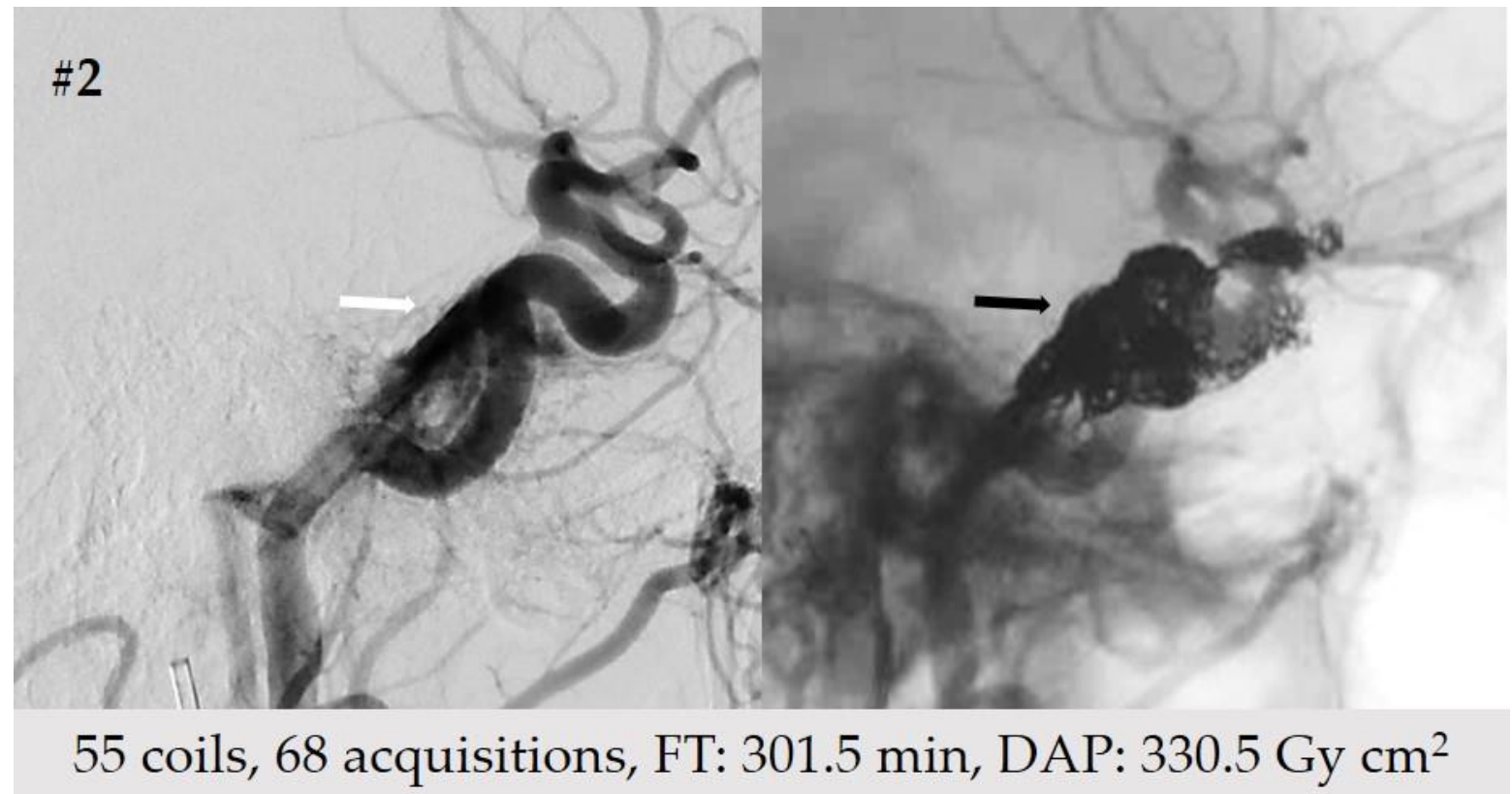

Figure 3. Dosimetrics of two exemplary patients with indirect fistulas. Patient \#1 was endovascularly treated with the lowest $(n=9)$ and patient \#2 with the highest number of coils $(n=55)$ in our study cohort. White arrows point to early filling of the cavernous sinus with contrast. Black arrows indicate implanted coils within the fistulous compartment.

\section{Conclusions}

The present study provides novel local DRLs in the field of endovascular treatment of CCFs using detachable coils, the most established and durable therapy option. Procedural radiation exposure was significantly higher in patients with indirect fistulas when compared to direct fistulas. Regarding radiation dose optimization, the application of a dedicated LD DSA protocol allowed for a $32.6 \%$ DAP reduction.

Author Contributions: Conceptualization, Y.O. and R.F.; methodology, Y.O. and R.F.; formal analysis, Y.O.; writing—original draft preparation, Y.O. and R.F.; writing—review and editing, C.G.T., R.S., T.L., R.F. All authors have read and agreed to the published version of the manuscript. 
Funding: This research received no external funding.

Institutional Review Board Statement: The study was conducted in accordance with the Declaration of Helsinki, and approved by the Ethics Committee of Ludwig-Maximilians-University (project number: 20-0450, date of approval: 3 February 2022).

Informed Consent Statement: Patient consent was waived due to retrospective nature of the study.

Data Availability Statement: Not applicable.

Conflicts of Interest: The authors declare no conflict of interest.

\section{References}

1. Barrow, D.L.; Spector, R.H.; Braun, I.F.; Landman, J.A.; Tindall, S.C.; Tindall, G.T. Classification and treatment of spontaneous carotid-cavernous sinus fistulas. J. Neurosurg. 1985, 62, 248-256. [CrossRef] [PubMed]

2. Ringer, A.J.; Salud, L.; Tomsick, T.A. Carotid cavernous fistulas: Anatomy, classification, and treatment. Neurosurg. Clin. N. Am. 2005, 16, 279-295. [CrossRef] [PubMed]

3. Korkmazer, B.; Kocak, B.; Tureci, E.; Islak, C.; Kocer, N.; Kizilkilic, O. Endovascular treatment of carotid cavernous sinus fistula: A systematic review. World J. Radiol. 2013, 5, 143-155. [CrossRef] [PubMed]

4. Ertl, L.; Brückmann, H.; Patzig, M.; Fesl, G. Endovascular therapy of direct dural carotid cavernous fistulas-A therapy assessment study including long-term follow-up patient interviews. PLoS ONE 2019, 14, e0223488. [CrossRef]

5. Ertl, L.; Brückmann, H.; Patzig, M.; Dorn, F.; Fesl, G. Patient reported long-term outcome after endovascular therapy of indirect dural carotid cavernous fistulas. PLoS ONE 2020, 15, e0231261. [CrossRef] [PubMed]

6. Yoshida, K.; Melake, M.; Oishi, H.; Yamamoto, M.; Arai, H. Transvenous embolization of dural carotid cavernous fistulas: A series of 44 consecutive patients. AJNR Am. J. Neuroradiol. 2010, 31, 651-655. [CrossRef]

7. $\quad$ Bink, A.; Goller, K.; Lüchtenberg, M.; Neumann-Haefelin, T.; Dützmann, S.; Zanella, F.; Berkefeld, J.; du Rochemont, R.D.M. Long-term outcome after coil embolization of cavernous sinus arteriovenous fistulas. AJNR Am. J. Neuroradiol. 2010, 31, 1216-1221. [CrossRef]

8. $\quad$ Alexander, M.D.; Halbach, V.V.; Hallam, D.K.; Cooke, D.L.; Ghodke, B.V.; Dowd, C.F.; Amans, M.R.; Hetts, S.W.; Higashida, R.T.; Meyers, P.M. Long-term outcomes of endovascular treatment of indirect carotid cavernous fistulae: Superior efficacy, safety, and durability of transvenous coiling over other techniques. Neurosurgery 2019, 85, E94-E100. [CrossRef]

9. Chi, C.T.; Nguyen, D.; Duc, V.T.; Chau, H.H.; Son, V.T. Direct traumatic carotid cavernous fistula: Angiographic classification and treatment strategies. Study of 172 cases. Interv. Neuroradiol. 2014, 20, 461-475. [CrossRef]

10. Alexandre, A.M.; Sturiale, C.L.; Bartolo, A.; Romi, A.; Scerrati, A.; Flacco, M.E.; D'Argento, F.; Scarcia, L.; Garignano, G.; Valente, I.; et al. Endovascular treatment of cavernous sinus dural arteriovenous fistulas. Institutional series, systematic review and meta-analysis. Clin. Neuroradiol. 2021. online ahead of print. [CrossRef]

11. Federal Ministry of Justice and Consumer Protection. Regulation on Radiation Protection; Federal Ministry of Justice and Consumer Protection: Berlin, Germany, 2018. Available online: http:/ / www.gesetze-im-internet.de/strlschv_2018 (accessed on 17 January 2022). (In German)

12. Bundesamt für Strahlenschutz. Diagnostic Reference Levels. Available online: http://www.bfs.de/DE/themen/ion/anwendungmedizin/diagnostik/referenzwerte/referenzwerte_node.html (accessed on 17 January 2022).

13. Forbrig, R.; Ozpeynirci, Y.; Grasser, M.; Dorn, F.; Liebig, T.; Trumm, C.G. Radiation dose and fluoroscopy time of modern endovascular treatment techniques in patients with saccular unruptured intracranial aneurysms. Eur. Radiol. 2020, 30, $4504-4513$. [CrossRef]

14. Forbrig, R.; Stahl, R.; Geyer, L.L.; Ozpeynirci, Y.; Liebig, T.; Trumm, C.G. Radiation dose and fluoroscopy time of endovascular treatment in patients with intracranial lateral dural arteriovenous fistulae. Clin. Neuroradiol. 2021, 31, 1149-1157. [CrossRef] [PubMed]

15. Ozpeynirci, Y.; Trumm, C.; Stahl, R.; Fischer, D.; Liebig, T.; Forbrig, R. Radiation dose and fluoroscopy time of diagnostic angiography in patients with spinal dural arteriovenous fistula. Clin. Neuroradiol. 2022. online ahead of print. [CrossRef] [PubMed]

16. Opitz, M.; Alatzides, G.; Zensen, S.; Bos, D.; Wetter, A.; Guberina, N.; Oppong, M.D.; Wrede, K.H.; Hagenacker, T.; Li, Y.; et al. Radiation exposure during diagnostic and therapeutic angiography of carotid-cavernous fistula: A retrospective single center observational study. Clin. Neuroradiol. 2021. online ahead of print. [CrossRef] [PubMed]

17. Vañó, E.; Miller, D.L.; Martin, C.J.; Rehani, M.M.; Kang, K.; Rosenstein, M.; Ortiz-López, P.; Mattsson, S.; Padovani, R.; Rogers, A. ICRP Publication 135: Diagnostic reference levels in medical imaging. Ann. ICRP 2017, 46, 1-144. [CrossRef] [PubMed]

18. Lee, M.Y.; Kwon, J.; Ryu, G.W.; Kim, K.H.; Nam, H.W.; Kim, K.P. Review of national diagnostic reference levels for interventional procedures. Prog. Med. Phys. 2019, 30, 75-88. [CrossRef]

19. European Commission Radiation Protection $\mathrm{N}^{\circ}$ 180. Diagnostic Reference Levels in Thirty-Six European Countries Part 2/2; European Commission: Luxembourg, 2014. 
20. Australian Radiation Protection and Nuclear Safety Agency. Current Australian National Diagnostic Reference Levels for Image Guided and Interventional Procedures. Available online: https://www.arpansa.gov.au/research-and-expertise/surveys/ national-diagnostic-reference-level-service/current-australian-drls/igip (accessed on 17 January 2022).

21. Opitz, M.; Zensen, S.; Bos, D.; Li, Y.; Styczen, H.; Wetter, A.; Guberina, N.; Jabbarli, R.; Sure, U.; Forsting, M.; et al. Radiation exposure in the endovascular therapy of cranial and spinal dural arteriovenous fistula in the last decade: A retrospective, single-center observational study. Neuroradiology 2021, 64, 587-595. [CrossRef]

22. Kien, N.; Rehel, J.-L.; Étard, C.; Aubert, B. Dose patient en neuroradiologie interventionnelle: Bilan d'une enquête multicentrique [article in French]. J. Radiol. 2011, 92, 1101-1112. [CrossRef]

23. Guenego, A.; Mosimann, P.J.; Pereira, V.M.; Nicholson, P.; Zuber, K.; Lotterie, J.A.; Dobrocky, T.; Marcellus, D.G.; Olivot, J.M.; Piotin, M.; et al. Proposed achievable levels of dose and impact of dose-reduction systems for thrombectomy in acute ischemic stroke: An international, multicentric, retrospective study in 1096 patients. Eur. Radiol. 2019, 29, 3506-3515. [CrossRef]

24. Söderman, M.; Mauti, M.; Boon, S.; Omar, A.; Marteinsdóttir, M.; Andersson, T.; Holmin, S.; Hoornaert, B. Radiation dose in neuroangiography using image noise reduction technology: A population study based on 614 patients. Neuroradiology 2013, 55, 1365-1372. [CrossRef]

25. van der Marel, K.; Vedantham, S.; van der Bom, I.M.J.; Howk, M.; Narain, T.; Ty, K.; Karellas, A.; Gounis, M.J.; Puri, A.S.; Wakhloo, A.K. Reduced patient radiation exposure during neurodiagnostic and interventional X-ray angiography with a new imaging platform. AJNR Am. J. Neuroradiol. 2017, 38, 442-449. [CrossRef] [PubMed]

26. Kahn, E.N.; Gemmete, J.J.; Chaudhary, N.; Thompson, B.G.; Chen, K.; Christodoulou, E.G.; Pandey, A.S. Radiation dose reduction during neurointerventional procedures by modification of default settings on biplane angiography equipment. J. Neurointerv. Surg. 2016, 8, 819-823. [CrossRef] [PubMed]

27. Miller, D.L.; Kwon, D.; Bonavia, G.H. Reference levels for patient radiation doses in interventional radiology: Proposed initial values for U.S. practice. Radiology 2009, 253, 753-764. [CrossRef] [PubMed] 